\title{
Survey of Astronomical Image Processing Methods
}

\author{
Hai Jing Zhu, Bo Chong Han, and Bo Qiu ${ }^{(\bowtie)}$ \\ School of Electronic and Information Engineering, \\ Hebei University of Technology, Tianjin, China \\ sfighter@126.com
}

\begin{abstract}
The image processing is becoming a key issue in astronomical data analysis. This paper introduces the algorithms and methods applied in astronomical image processing from different aspects. The paper first shows methods of lossless compression of astronomical images, like the pyramidal median transform, segment-based DPCM and 5/3 integer wavelet transform. Secondly it shows the algorithms of astronomical image segmentation, edge detection and de-noising. Finally, many different methods of image recovery and restoration are introduced briefly. We summarize a number of recent achievements on astronomical image processing in this survey, and list the recent published papers.
\end{abstract}

Keywords: Astronomical image $\cdot$ Image compression $\cdot$ Image restoration $\cdot$ Survey

\section{Introduction}

With the development of astronomy, image processing has become more and more important and played a key role for astronomical research.

Raw astronomical images usually contain noises and blurs, so that they cannot be used in analysis and research directly. And under the enhancement of quality, the size of astronomical image grows up. As a result, it is difficult to store and transmit these large image files. Consequently, image processing in astronomy such as compression, denoising, segmentation and restoration is required.

In this paper we give a general review of astronomical image processing methods. Our review starts from Sect. 2 with image compression problems. In Sect. 3 we consider the issue of image segmentation and edge detection. In Sect. 4 the deblurring and denoising filtering methods are reviewed. The algorithms of image restoration are described in Sect. 5. We will review a range of important results in astronomical image processing, and a central theme about how these methods and algorithms are put forward and how they work.

\section{Astronomical Image Compression}

With the development of technology, image has been becoming a more and more critical medium of information. However, when we begin to study astronomical images, we find that the high-quality images are always with a large size and carry large 
databases. So it is interesting for the data providers and users to make use of the most effective image compression techniques.

Image compression is required in many fields like medicine, remote sensing and astronomy. Compression reduces the storage media costs and the network bandwidth needed to transmit the files to users. Image compression also reduces a number of bytes of data that are transferred to or from local disks during data analysis operations. In [8], we know the extensive literature on astronomical image compression can be divided into 2 main categories: loss compression, in which some of the information (ideally only noise) is discarded, and lossless compression techniques where all the information is preserved so that the original data can be exactly reconstructed. Lossless compression by definition preserves all the information in the images and is often preferred or required in situations where the data provider must be certain that no information is lost. In this paper we just focus on lossless compression.

Traditional methods of lossless compression like Huffman coding, arithmetic coding, dictionary based LZW encoding, bit plane coding and the run length encoding widely used in different situations. The Huffman coding method based on statistical properties of image is used in star image where the most areas are stable; while the bit-plane coding method is more available in images with many details or with a high resolution, for instance the photographs of the sun.

The wavelet transform is also extensively used to astronomical image compression. In [5], it presents an algorithm based on the wavelet transformation which can achieve image compression with or without information loss, as well as compression of specific interesting image regions. The paper focuses on the compression encoding technique after wavelet transformation, when finished the analysis of frequency band the critical parameters are extracted to transmit so that this algorithm can obtain a good result of image information with a least data. According to the fluctuations in network bandwidth the algorithm timely adjusts the compression rate. The astronomical image information will be effectively transmitted for remote observation in time.

In [8], the author uses a relatively new compressed image format that is based on the FITS tiled-image compression convention. The current implementation of this convention in the CFITSIO library supports 4 lossless compression algorithms: Rice, Hcompress, PLIO and GZIP. The article introduces the main features of each of the algorithms. It should be noted that the GZIP algorithm has a users electable parameter for fine tuning the tradeoff between speed and compression ratio. Also the effect of noise on lossless image compression is discussed and a comparison on different compression algorithms is made. In this paper the author has performed a detailed evaluation of various lossless compression techniques on a large sample of astronomical images that are representative of the types of images from many sky survey projects. Using optimal compression methods on such images can make a large difference in the amount of disk space and network bandwidth needed to analyze, archive, and reconstruct the images.

In [11], the paper describes the basis theoretical of image compression, given a detailed description of the implementation principle of Huffman coding, LZW coding and predictive coding. Then it introduces the wavelet transform theory, expounds the second generation of integer wavelet transform theory and implementation steps based on lifting scheme, compares these technologies with experimental methods, and points 
out that an important prospects for hybrid coding. The characteristics of astronomical images are analyzed and summarized: large, special, formatted, high-gray and noisy. In order to solve the high-gray problem, this paper uses gray image stretching technology to achieve astronomical images display. After analyzing the experiment results of predicting and IWT, this paper proposes a new sub-block DPCM and integer wavelet transform using in astronomical image lossless compression algorithm. And it has done a comprehensive exposition of the algorithm, then the importance of astronomical images sub-block is discussed in detail, while the DPCM prediction strategy is selected by experiments, and the $(5,3)$ wavelet is selected being symmetric extension of the wavelet transform boundary approach. The predicting and IWT algorithm applied in astronomical images is especially emphasized.

In [6], the paper presents a new lossless image compression method in order to solve the increasingly outstanding issue of storing massive astronomical image data in devices of limited storage space and low bandwidths [3]. In [1], the author uses integer 5/3 wavelet transformation with the combination of DPCM to achieve a lossless compression of image, however, the compression ratio is not so good since the Haar wavelet has a weak ability of redundancy removing. In [4], the authors combine DPCM with IWT and get a lossless compression of medical images with a higher compression ratio, but, when the picture is extra large this method will arouse a diffusion of prediction error. In [7], the loss and lossless image compression methods are improved by using EZW, but the algorithms are too complex to achieve hardware implementation. In the method presented in [6] a large astronomical image is segmented first, then successively coded with the Differential Pulse Code Modulation, 5/3 Integer Wavelet Transformation, and Huffman algorithm. The author briefly outlines the approach of the lossless compression, and discusses the details of the principle of the method and the implementation of the relevant algorithms. The experiment indicates that the compression ratio of out method is higher than those of the Linux tar command, PKZip, WinZip, and WinRar, respectively; the compression ratio is also much faster than the software's WinZip and WinRar. In addition, this algorithm has a parallel structure and is easy for hardware implementation.

\section{Image Segmentation and Edge Detection}

In astronomy, researchers aren't commonly interested in all the information in an image when they are studying on it; on the contrary, they often pay more attention to some regions of an image, which are generally called foreground. So it is very important to delete unnecessary data when memorizing and transmitting huge data [12]. One extensively used approach is, according to the characteristics of astronomical images astronomers make edge detection by using LOG (Laplacian of the Gaussian) operator in order to find out the outline of foreground, then segment the foreground.

In [12] a view finding technology based on digital image processing method is introduced, which finds out where the interested region is and segment it by using LOG operator. Noticed that astronomical images are mostly with single foreground and pure background, there is large contract ratio between foreground and background and the edge of image is sharp so that the LOG operator can be efficient to detect the edge and segment the background. 
In astronomical image segmentation and edge detection, to meet the needs of high-speed and real-time is especially important. The paper [13] presents a method based on pipelining, using FPGA to build a SOC. Through design and verification it shows the method can achieve high speed, multi-channel and clock synchronization in astronomical image segmentation.

The paper [14] introduces four edge detection algorithms. They are different algorithms based on local hybrid filter de-noising model, sparse de-noising, least squares support vector machine, and the sparse representation de-noising model. In addition, the proposed algorithms are comparable, and their advantages and disadvantages in the SAR image edge detection are analyzed in order to facilitate further research.

In the sky, the stars' magnitudes vary a lot, so there are a lot of faint stars as shown in the star chart. When the distance between the imaging system and the target is big enough to a certain extent, the size of the target in the image is very small, may accounting for only a few, or even, a pixel. Meanwhile, the energy due to the attenuation caused by long-distance transmission, the star source signal intensity is relatively low with respect to the noise. Under such conditions, to detect the moving point target has important applications in guidance, astronomical observations, remote sensing and other disciplines.

(1) In the case of low SNR, it is not a small problem to detect moving point targets. For detecting moving point targets, in order to avoid the exhaustive search for the three-dimensional image sequences to detect moving targets, researchers have explored a number of ways, including 'a matched filter method' (1983, Reed), which uses matched filter theory applied to the three-dimensional image processing sequence, where the moving point target detection problem is transformed into a three-dimensional transform domain to find the optimal (matched) filter problem. And besides of this, there are dynamic programming method, Hove transform optimal projection method, sequential hypothesis testing, neural networks and other methods.

(2) With the development of photonics technology, astronomy and navigation technology has been improved in celestial navigation system for automatic generation of artificial sight detection technology, which is becoming increasingly important. It has become a powerful tool to improve the accuracy. Astral detection unit is a key part of celestial navigation system, which directly detects the position of the stars in the field of star tracking and direct astronomical positioning. Gray distribution feature uses satellite target point by sub-pixel positioning algorithm to determine the position of the target image. Sub-pixel positioning algorithms which can be used here are: centroid, weighted centroid method, Gaussian surface fitting method, and parabolic fitting method.

(3) In [15-17], star pattern recognition algorithms are introduced. Star sensor key algorithm, is to establish the relationship between the stars in star map and satellite image points according to the inertia coordinate system, in order to gain information about the location of the reference star. Star pattern recognition is a more mature star sensor algorithm to get the star map of each reference star magnitude, location coordinates and other information through the star identification. 
Currently star pattern recognition algorithm can be applied in practical engineering, to the principle of a simple, easy to implement to identify triangles majority. Though there are some flaws in triangle recognition algorithms, domestic and foreign researchers are still improving it to achieve high recognition hit rate.

Figure 1 shows astronomical positioning triangle: $\mathrm{O}$ is the station, $\mathrm{Z}$ is the zenith, $\mathrm{P}$ is the north celestial pole. Guide device is based on the visual axis in the geocentric inertial coordinate system, given astronomical latitude and longitude respectively. By location triangle, horizontal photoelectric measuring system can work properly.

By the star pattern recognition to determine the star map of the star image after correspondence with the stars, it can be based on the principle of pinhole imaging model. Pinhole imaging model is shown in Fig. 2.

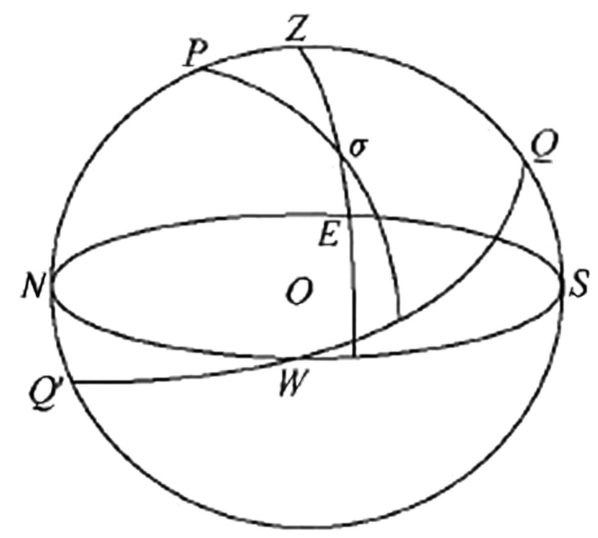

Fig. 1. Positioning the triangle diagram of astronomy

\section{Denoising}

In astronomical research, the images are often obtained by CCD (Charge-Coupled Device). Those images are normally affected by all kinds of noises during acquisition and transmission. And due to the interference of cosmic rays, some 'salt noises' can systematically introduce into CCD images during their acquisition. It is necessary to study how to reduce the noises effectively and how to realize the star searching and centering.

In [18], a ROAD (Rank-Ordered Absolute Difference) algorithm used to remove impulse noises and stellar-searching in CCD image is systematically introduced. The ROAD statistical values quantify how different in intensity the particular pixels are from their most similar neighbors. The noise removal filter based on a simple statistic to detect impulse pixels is to effectively remove noise from an image while keeping its features intact.

In [19], the author proposes a new parametric imaging technique which improves the resolution and sensitivity by enhancing the MVDR parametric imaging and 


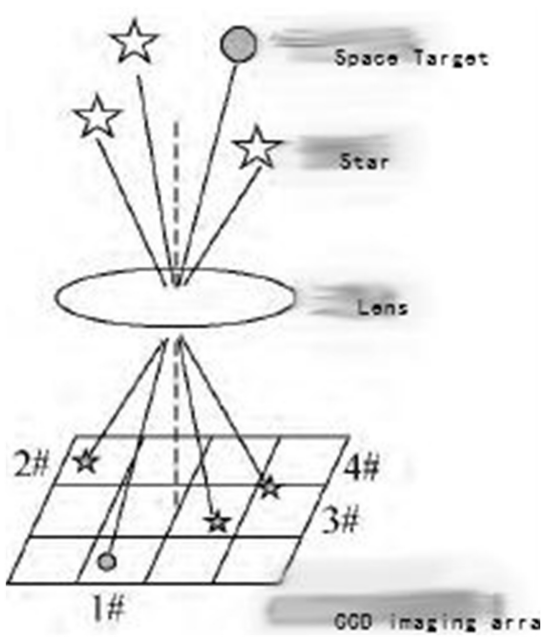

Fig. 2. Schematic model of pinhole imaging

introducing better power estimates based on least squares. Simulations demonstrate that the approach has higher resolution, and performs better in noisy situations.

In [14], several de-noising models based on the multi-scale geometric transformation have been proposed for the imaging features of SAR image, including the cycle spinning de-noising model based on Wavelet Contourlet transform, Gaussian mixture de-noising model based on the complex Contourlet transform, de-noising model based on local hybrid filter, and so on.

\section{Restoration and Reconstruction}

In most applications, the acquired images represent a degraded version of the original scene for instance astronomical imaging (e.g., using ground based imaging systems or extraterrestrial observations of the earth and the planets). The degradation can be due to the atmospheric turbulence, the relative motion between the camera and the scene, and the finite resolution of the acquisition instrument. So image restoration is a key issue in astronomy. However image restoration is a well-known, ill-posed inverse problem which requires regularization.

\subsection{Reconstruction from Speckle Image}

Because of atmospheric turbulence, the performance of high resolution imaging of large optical instruments is severely limited. Speckle imaging is one of the ways to eliminate atmospheric blurring based on recording series of images with short exposure to freeze the turbulence to obtain restoration image for reconstruction. Recently speckle image reconstruction has been shown great power to help binary star system in related reports. 
In [25], the paper gives a review of how astronomical images are reconstructed from speckle images. The methods of speckle image reconstruction are described, including methods in both frequency domain and spatial domain. In the frequency part of the article, speckle interferometer for retrieving modulus information is introduced. Knox-Thompson, speckle masking and phase reconstruction from modulus for retrieving phase information are also introduced in the frequency part. LWH method and iterative shift-and-add method are introduced in the spatial part. Not only the methods of astronomical image reconstruction themselves but also their applications are presented in the paper.

In [26], a modified maximum likelihood blind de-convolution algorithm is proposed in this paper by incorporating dynamic shrinkage of support region. The algorithm is applied to the restorations of short exposure images of astronomical point source star, images of astronomical extended object and adaptive optics long exposure images of point source star. Experimental results prove that the algorithm works well not only for short exposure images of point source star, but also for images of astronomical extended object and adaptive optics long exposure images. The PSNR of reconstruction images is raised by over $10 \%$. The novelty of this algorithm is that the support region of astronomical object can be contracted adaptively according to the threshold in reconstruction iterations.

In [27], the author surveys image reconstruction methods aimed at speckle images and methods to simulate the speckle images. Then the author tests typical reconstruction methods and introduces those methods in his dissertation. On the other hand a reconstruction method based on Kalman filter and containing physical constraints is proposed and tested with both simulated and real data. While to contrast with it the Kalman filter based reconstruction method without physical constraints is also illustrated. The Kalman filter based reconstruction with physical constraints shows positive prospect.

\subsection{Blind Deconvolution Methods}

In astronomy, image resolution and contrast will be decreased because of the complexity of imaging conditions. When we observe an astronomical object from the surface of the earth, its emitted or reflected light has to invariably pass through the atmosphere, which yields a blurry observed image. De-blurring images of an observed celestial body is therefore a fundamental problem in astronomy. This problem is compounded by the fact that the blur is not only unknown, but also is continually changing in time as well as spatially due to refraction-index fluctuations caused by atmospheric turbulence. So image restoration has being a study focus in image processing. Existing image restoration algorithms, such as Wiener filtering, builds on the known point spread function. However, point spread function is often unknown in practice because imaging conditions are unknown, like the relative motion between camera and objects and atmospheric disturbance. Therefore, the research on blind de-convolution and blind restoration is very necessary in theory and practice.

The all-pervasive presence of noise is what makes de-convolution particularly difficult. The paper 'De-convolution in Astronomy: a Review' gives a review of different de-convolution methods in recent 10 years. The diversity of resulting algorithms 
reflects different ways of estimating the true signal under various idealizations of its properties. Different ways of approaching signal recovery are based on different instrumental noise models, whether the astronomical objects are point-like or extended, and indeed on the computational resources available to the analysts. It presents a number of results in the survey of image restoration.

In the paper [34], a blind de-convolution approach based on the maximumlikelihood estimation technique with real optical band limitation constraint is presented for removing the effect of atmospheric turbulence on this class images through the minimization of the convolution error function by using the conjugation gradient optimization algorithm. As a result, the object function and the point spread function could be estimated from a few record images at the same time by the blind de-convolution algorithm. According to the principle of Fourier optics, the relationship between the telescope optical system parameters and the image band constraint in the frequency domain is formulated during the image processing transformation between the spatial domain and the frequency domain.

The convergence property of multi-frame blind de-convolution applied to improve the resolution of astronomical image is influenced by initial object estimate, constraint conditions and photon noise. In the paper [36], a method of determining band limitation of the point-spread function from the optical imaging system parameters is proposed, in which the initial object estimate of blind de-convolution algorithm is formed by using Knox-Thompson method to reconstruct object phase from short-exposure images. A novel method, which is used to decrease photon noise, edge effects and ringing of restoration images, is developed. An improved multi-frame blind de-convolution algorithm with a strict constrained optimization method of the expectationmaximization based on maximum likelihood estimation is presented. The restored images in experiment show that the proposed multi-frame blind de-convolution algorithm is valid for overcoming effects of atmospheric turbulence and photon noise, improving the resolution of real observed astronomical images, and capable of partly removing the diffraction effect of optical system on restored images.

\subsection{Restoration Based on Maximum-Likelihood Algorithm}

Traditional image restoration algorithms always deal with Gaussian noise, however, the real astronomical images are polluted by Gaussian and Poisson mixed noise. Therefore, the paper [28] introduces a imaging model of Poisson-Gaussian distribution, makes an effective approximation to the Maximum Likelihood (ML) algorithm based on the mixed model, and proposes a modified ML algorithm based on Poisson-Gaussian mixed noise to avoid the sensitivity to noise and the dependence to the original estimation of PSF. Experimental results show that the algorithm works well, and the robustness is well.

In [29], the effective approximation model based on Maximum-likelihood (ML) function proposed by Benvenuto is analyzed for astronomy image restoration, then a new image restoration algorithm with convergence faster than that of traditional ML method is proposed in which PSF known a prior is not required. The turbulence PSF is estimated from observed blur images to make the PSF estimation in accordance with an imaging environment. By incorporating adaptive estimation of PSF into ML 
restoration, an enhanced ML algorithm is presented. Additionally, the PSF is updated successively during iteration. The ML restoration and de-noising are performed alternatively in iteration. The results show that the proposed algorithm works much better than ML does. In conclusion, the algorithm allows the iterative process in ML algorithm to converge stably and the image quality to be improved. Experiment results show that the presented method can be used routinely in astronomical image restoration.

\section{Conclusion}

In this study we give a review of the astronomical image processing methods and sort them into different aspects. In this field, it has a lot of special issues, as well as the common issues in image processing field. They may influence each other.

\section{References}

1. Tian, J., Liu, B., Liu, J.: The information entropy preserved coder of still image by integer haar wavelet transforms and sub block DPCM. J. China Inst. Commun. 11, 29-34 (2000)

2. Li, Z., Zhou, W.: The application of the digital image compression technique to astronomy. Prog. Astron. 14, 259-261 (1996)

3. Zhao, L., Tian, Y., Sha, Y., Li, J.: Lossless compression of medical images by combining integer wavelet transform with DPCM. J. Northeast. Univ. (Nat. Sci.) 10, 1454-1457 (2007)

4. Wu, D., Wang, J., Zhang, H.: Research of hyper-spectral image lossless compressing technology based on DPCM between spectra and IWT. Acta Photonica Sin. 1, 156-159 (2008)

5. Ye, H., Dong, S.: Satellite cloud imagery compression based on wavelet transform. Comput. Eng. Sci. 10, 60-65 (2004)

6. Guifu, Z., Hui, D., Feng, W., Kaifan, J.: Study of a lossless compression method of astronomical images using segment-based DPCM and 5/3 integer wavelet transformations. Astron. Res. Technol. 1, 73-80 (2011)

7. Li, L., Dai, H.B., Xu, J.: The study of the application of image compression based on wavelet transformation in astronomical remote observation. Astron. Res. Technol. 4, 380 384 (2008)

8. Pence, W.D., Seaman, R., White, R.L.: Lossless astronomical image compression and the effects of noise. Publ. Astron. Soc. Pac. 121, 414-427 (2009)

9. Haixia, C.: The research of lossless compression arithmetic based on integer wavelet transform's still image. Xi'an University of Science and Technology, Xi'an (2008)

10. Shengxue, Q.: Satellite image processing algorithm research and its corresponding hardware implementation. Beijing University of Posts and Telecommunications, Beijing (2007)

11. Guifu, Z.: Research and realization of lossless compression methods of astronomical image. Kunming University of Science and Technology, Kunming (2009)

12. Meng, K., Jin, S.-Z., Wang, B., Wang, Y.: Viewfinding technology in astronomic images. Res. Comput. Appl. 72-73, 101 (2003)

13. Fei, W., Tang, Q., Liu, P., Cai, H.: Implementation of high-speed astronomical image segmentation based on pipelining. Sci. Technol. Eng. 22, 5433-5436 (2010)

14. Shuaiqi, L.: The processing algorithm study for remote sensing images based on multi-scale geometric transformation. Beijing Jiaotong University, Beijing (2013) 
15. Bo, Z.: Independent study day hierarchical star identification algorithm based on star sensor. Graduate School of Chinese Academy of Sciences (Changchun Institute of Optics and Precision Instruments and physical), Changchun (2004)

16. Lei, Z., Xin, H., Zhonghui, W.: Other fast-sky autonomous star identification. Optics and Precis. Eng. 17(4), 909-915 (2009)

17. Zhang, S.D., Wang, Y., Sun, H.: Triangulation and radial basis function neural network in star pattern recognition. Optics Precis. Eng. 20(2), 395-402 (2012)

18. Wenzhong, X.: Design and implementation of denoising and star-searching in ccd astronomical images. Jinan University, Guangzhou (2007)

19. Ben-David, C., Leshem, A.: Parametric high resolution techniques for radio astronomical imaging. IEEE J. Sel. Top. Sig. Proc. 2, 670-684 (2008). Bar-Ilan University, Ramat Gan

20. Yubao, C.: Research of infrared weak-target detection based on mathematical morphology, pp. 322-326, Hefei (2006)

21. Han, J., Zhang, Y., Chen, Z.: Real time detection algorithm of weak targets in astronomical images. Opto-Electron. Eng. 12, 1-4 (2005)

22. Jingchang, A.: Research on automated processing and target detection of astronomical. Information Shandong University, Jinan (2011)

23. Chantas, G.K., Galatsanos, N.P., Likas, A.C.: Bayesian restoration using a new nonstationary edge-preserving image prior Giannis K. Chantas. IEEE Trans. Image Process. 15(10), 2987-2997 (2006)

24. Babacan, S.D., Molina, R., Katsaggelos, A.K.: Parameter estimation in tv image restoration using variational distribution approximation. IEEE Trans. Image Process. 17(3), 326-339 (2008)

25. Huo, Z., Zhou, J.: Methods of astronomical image reconstruction from speckle image. Prog. Astron. 1, 72-90 (2010)

26. Geng, Z., Wang, Z.: Modified high definition reconstruction algorithm of astronomical speckle images. Optics Precis. Eng. 7, 1151-1156 (2007)

27. Zhuoxi, H.: Research on astronomical image reconstruction from speckle image. Tsinghua University, Beijing (2010)

28. Wei, X., Geng, Z., Song, X., Wang, L., Tang, C.: Modified maximum likelihood algorithm based on Poisson-Gaussian mixed noise. Comput. Eng. 1, 222-224 (2012)

29. Geng, Z., Wei, X., Shen, C.: Astronomical image restoration based on maximum-likelihood incorporated parameter estimation. Optics Precis. Eng. 11, 2943-2950 (2013)

30. Vega, M., Mateos, J., Molina, R., Katsaggelos, A.K.: Astronomical image restoration using variational methods and model combination. Stat. Methodol. 9, 19-31 (2011)

31. Zhao, J., Wu, Y., Jin, J., Qiao, B., Wang, B., Wang, Z., Ma, X.: Image restoration based on real time wave-front information.Optics Precis. Eng. 6, 1350-1356 (2012)

32. Zhu, W., Sui, C., Tang, Y., Shen, X., Hu, J.: Application of restoration technology to blurred images in astronomical image processing. Infrared (Mon.) 3, 6-10 (2005)

33. Jichao, J., Baojun, Z., Fenfang, T., Juan, L.: Astronomical image enhancement algorithm based on regional image restoration. Chin. J. Sci. Instrum. 7, 1455-1461 (2011)

34. Bertero, M., Boccacci, P.: Image restoration methods for the large binocular telescope (LBT). Astron. Astrophys. 3, 1-15 (2000)

35. Luo, L., Wang, L., Cheng, W., Shen, M.: Approaches to convergence enhancement of multiframe blind deconvolution of astronomical images. Acta Phys. Sin. 12, 6708-6713 (2006)

36. Luo, L., Fan, M., Shen, M.: Blind deconvolution of astronomical images with band limitation determined by optical system parameters. Acta Astron. Sin. 3, 374-382 (2007) 